\title{
OBJECT-ORIENTED CHANGE DETECTION FOR REMOTE SENSING IMAGES BASED ON MULTI-SCALE FUSION
}

\author{
Wenqing Feng ${ }^{\text {a }}$, Haigang Sui ${ }^{\mathrm{a}, *}$, Jihui Tu ${ }^{\mathrm{a}, \mathrm{b}}$ \\ ${ }^{a}$ State Key Laboratory of Information Engineering in Surveying, Mapping and Remote Sensing, Wuhan University, \\ 430079 Wuhan, China (corresponding author, e-mail: haigang_sui@263.net) \\ ${ }^{\mathrm{b}}$ Electronics \& Information School of Yangtze University, Jingzhou, Hubei 434023,China
}

KEYWORDS: multi-scale segmentation; object; features; fuzzy fusion; decision level fusion;

\begin{abstract}
:
In the process of object-oriented change detection, the determination of the optimal segmentation scale is directly related to the subsequent change information extraction and analysis. Aiming at this problem, this paper presents a novel object-level change detection method based on multi-scale segmentation and fusion. First of all, the fine to coarse segmentation is used to obtain initial objects of different sizes; then, according to the features of the objects, Change Vector Analysis is used to obtain the change detection results of various scales. Furthermore, in order to improve the accuracy of change detection, this paper introduces fuzzy fusion and two kinds of decision level fusion methods to get the results of multi-scale fusion. Based on these methods, experiments are done with SPOT5 multi-spectral remote sensing imagery. Compared with pixel-level change detection methods, the overall accuracy of our method has been improved by nearly $10 \%$, and the experimental results prove the feasibility and effectiveness of the fusion strategies.
\end{abstract}

\section{INTRODUCTION}

Along with the rapid development of remote sensing data acquisition method and the gradual shortening of the acquisition cycle, its scope of application is becoming increasingly widespread and the application requirements are expanding. This presents higher requirements and challenges for remote sensing image change detection technology. At present, many research scholars at home and abroad use optical remote sensing image, according to different changing detection target, a lot of methods and effective models are proposed (Su et al.,2011; Hazel G et al.,2001; Dai et al.,2012; Liang et al.,2013;Zhong et al.,2005; Wang et al.,2013;Sun et al.,2010). These methods can be roughly divided into 3 categories: pixel-level change detection, feature-level change detection, object-level change detection (Hazel $\mathrm{G}$ et al., 2001). Traditional change detection methods mostly belong to the pixel level, the results of the change detection are generally broken, prone to the "salt and pepper phenomenon". These methods have higher requirements for the image registration accuracy and radiometric calibration. Simultaneously, relying on the threshold selection directly limit their application to remote sensing image processing. (Dai et al.,2012; Bovolo F et al.,2007; Bruzzone L et al.,2000; Nemmour $\mathrm{H}$ et al.,2006). However, the object level change detection is based on geographic objects that have some notion as a basis, these objects are spatially adjacent, spectrum similar in homogeneous areas, has the features of spectral, shape, texture, context, etc. (Wang et al., 2009; Wu et al., 2013; Su et al., 2007), it has high detection accuracy and robustness in the detection process. It represents the main direction of change detection.

At present, the object-level application of image analysis in change detection has seen certain progress. Many scholars apply object-oriented segmentation method to object-level change detection. Object-based change detection methods in common use can be divided into the following two categories. One is to carry out the object-oriented classification, and then change detection (Su et al., 2011; Hazel G et al., 2001; Liang et al., 2013). The other category is the object change vector analysis (Wang et al., 2013; Sun et al., 2010; Wu et al., 2013), which is a direct comparison method. Being easy to implement and having no essential difference with pixelbased approaches, the change vector analysis method has been widely used in practice/practically. The main difference lies in the pixel-level vector analysis being changed into the object-level vector analysis. Because the object of change vector analysis method can effectively utilize the various features of the object participation, such as spectral information, texture information, topology information between objects, etc., the result is more accurate and robust (Sun et al., 2010). However, the results of object-level change detection rely heavily on the determination of the segmentation scale. How to objectively evaluate the results of the segmentation, obtain optimal segmentation scale and avoid the influence of subjective factors are becoming particularly important (Chen et al., 2011).

Aiming at this problem, this paper presents a novel object-level change detection method based on multi-scale segmentation and fusion. To achieve the purpose, the following strategies are employed.

1) In order to obtain corresponding object, the two temporal images are unitedly segmented. Then multi-resolution segmentation method is used to extract objects. This step is called united segmentation.

2) Through choosing the optimal segmentation scale suitable for different objects, the OIF index is calculated of difference images on each scale. Then, the change intensity map is calculated based on object change vector analysis.

3) By comprehensively using the spectrum, and texture features of objects, and three different multi-scale fusing strategies, respectively, fuzzy fusion and different decision-level fusion are used to obtain the final detection results.

The rest of this paper is organized as follows. Section II describes the proposed method. Section III analyses and compares the experimental results, followed by the conclusion in Section IV.

\section{METHODOLOGY}

In order to reduce the influence of the difference of the radiation value of the optical image in different periods on the change detection accuracy, radiation correction is usually performed before the change detection. In this paper, the histogram matching method is used to carry out relative 
radiometric calibration (Wu et al., 2013). Then eCognition software is assisted to obtain the object through multi-scale segmentation. Then the segmentation result is regarded as the basis for change detection ( $\mathrm{Li}$ et al., 2011). By exploring the optimal segmentation scale suitable for different objects and to obtain appropriate scale values, the corresponding segmentation results with the two temporal images are achieved. Finally, the change intensity map of multiple scales based on spectral features and texture features is not only quantifying to achieve quantitative change intensity maps, but also through OTSU threshold segmentation to achieve the change binary maps. The fuzzy fusion and decision-level fusion methods are used to facilitate the integration of the change detection results obtained from different scales. Also, the test results including changes in position and changes include intensity information are obtained. The framework of the proposed method is shown below in Fig. 1.

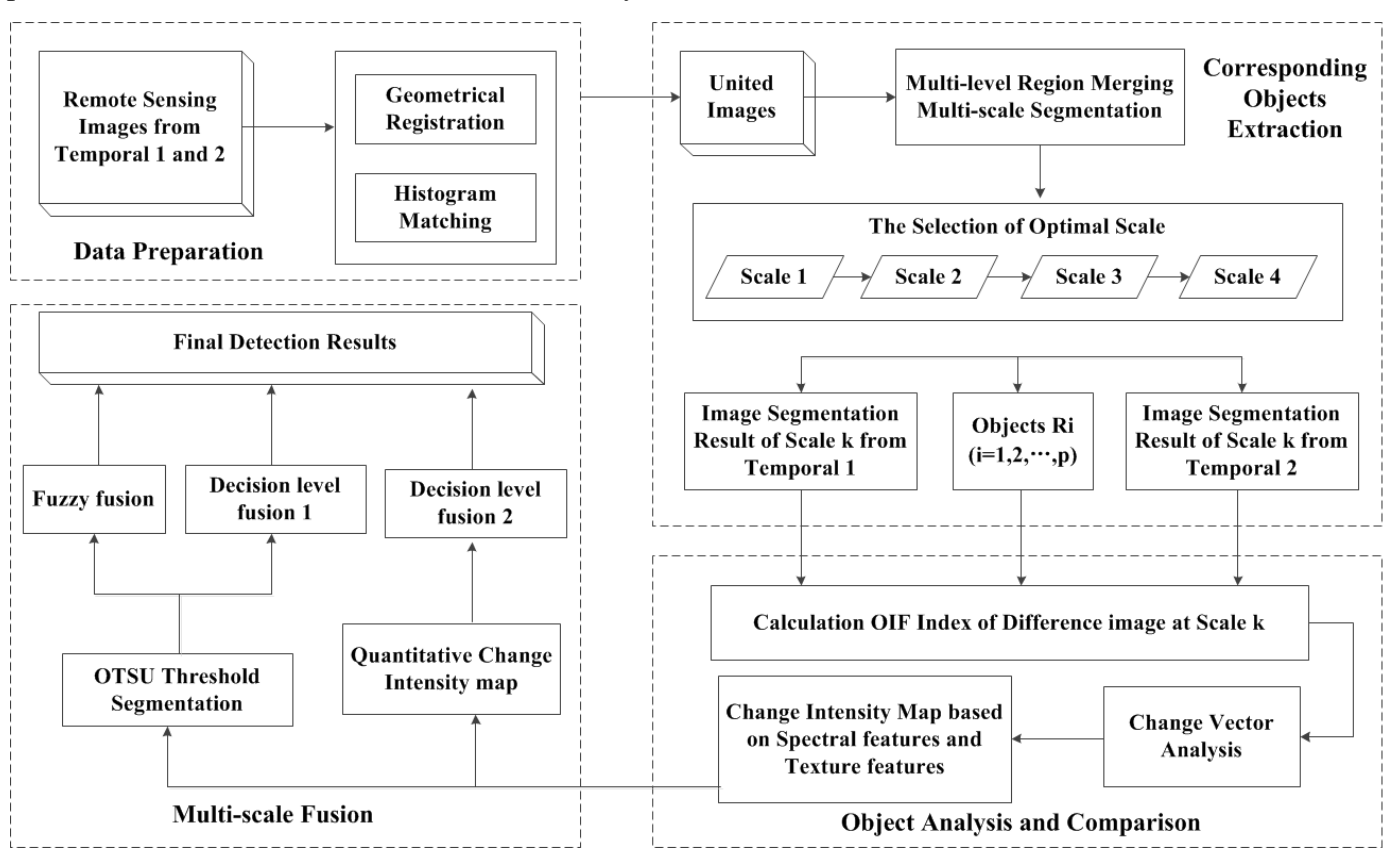

Figure.1 Framework of the proposed method

2.1 The difference calculation of spectral feature and texture feature

(1) Difference of spectral feature

The spectral information of an object mainly includes the mean value, standard deviation, ratio, brightness and other features. In this paper, the mean value and standard deviation of objects are used as difference metrics. The formula is as follows:

$$
\begin{gathered}
\text { diff _Mean_Band }(i)=H 1\left(x_{i}^{T_{1}}\right)-H 1\left(x_{i}^{T_{2}}\right) H 1 \text { :Mean } \\
\text { diff_Std_Band }(i)=H 2\left(x_{i}^{T_{1}}\right)-H 2\left(x_{i}^{T_{2}}\right) H 2: \text { Std }
\end{gathered}
$$

Where $\mathrm{i}$ is the number of image band.

(2) Difference of texture feature

According to GLCM (Gray-level Co-occurrence Matrix), Haralick and other researchers, defined 14 kinds of texture features, which are often used to extract texture information in remote sensing image, is mean value, standard deviation, contrast, entropy, homogeneity, correlation, angular second moment, dissimilarity etc. In the above texture features, texture entropy is the measure of the image information. It is characterized by the complexity of the image texture, and the more complex the image texture, the larger the entropy value, the less complex the image texture, the smaller the entropy value. Under the comprehensive of effectiveness and robustness, texture entropy is used to evaluate texture differences. The calculation formula is as follows:

diff_ent_Band $(i)=H 3\left(x_{i}^{T_{1}}\right)-H 3\left(x_{i}^{T_{2}}\right) H 3$ : Entropy

Where $\mathrm{i}$ is the number of image band.

\subsection{Best Band Selection}

The standard deviation and the correlation coefficient between different bands are the best indicators in measuring the amount of information and correlation, and are commonly used in calculations; the formula of optimal band combination is

$$
O I F=\frac{\sum_{i=1}^{N} \sigma_{i}^{f}}{\sum_{i=1}^{N} \sum_{j=i+1}^{N}\left|R_{i j}^{f}\right|}
$$

This formula is the best band index-OIF index. By extracting the mean value of each band on the spectrum, standard deviation difference chart, texture entropy difference chart, and calculating their standard deviation in each band $\sigma_{i}^{f}$, corresponding to the three features above; where $R_{i j}^{f}$ is the correlation coefficient of $\mathrm{f}$ feature differences graph between the band diagram $\mathrm{i}$ and $\mathrm{j}$, and $\mathrm{N}$ is the total number of bands (Liang et al., 2012).This approach was proposed by the American scholar Richard Horowitz. Its theoretical basis is: The larger the standard deviation of the data in the band combination is, the more information it contains; However, the smaller the correlation coefficient is, which shows the independence of the data is higher, the redundancy of information is smaller, it effectively unites standard deviation and correlation coefficients, providing a basis for judging the best combination of bands (Wu et al., 2007).

In this paper, OIF index is applied to find the possible combination of the spectrum mean value, standard deviation and entropy texture feature bands to discover the appropriate wave band combination separately, and based on the principle of maximum OIF index to find the best band combination of spectrums and texture difference. 


\subsection{Fuzzy fusion analysis}

First, we determine the change intensity observation value $Y_{k}(i, j)$ of the change intensity map on the fourth scales $Y_{k}$ in relation to the change $\mu_{c}\left(\mathrm{Y}_{k}(i, j)\right)$ of degrees category $w_{c}$ and with respect to the membership $\mu_{u}\left(\mathrm{Y}_{k}(i, j)\right)$ of the non-change category $w_{u}$. The common $\mathrm{S}$ type membership function has robustness and accuracy in blurred image segmentation process (Tobias et al., 2011). Therefore, the following function is used to calculate the membership value of $\mu_{c}\left(\mathrm{Y}_{k}(i, j)\right)$, and the formula is as follows:

$$
\mu_{c}\left(Y_{k}(\mathrm{i}, \mathrm{j})\right)=\left\{\begin{array}{cc}
0, & Y_{k}(\mathrm{i}, \mathrm{j}) \leq a_{k} \\
2\left(\frac{Y_{k}(\mathrm{i}, \mathrm{j})-a_{k}}{c_{k}-a_{k}}\right)^{2}, & a_{k}<Y_{k}(\mathrm{i}, \mathrm{j}) \leq b_{k} \\
1-2\left(\frac{c_{k}-Y_{k}(\mathrm{i}, \mathrm{j})}{c_{k}-a_{k}}\right)^{2}, & b_{k}<Y_{k}(\mathrm{i}, \mathrm{j}) \leq c_{k} \\
1, & Y_{k}(\mathrm{i}, \mathrm{j})>\mathrm{c}_{k}
\end{array}\right.
$$

Where $b_{k}=\left(a_{k}+c_{k}\right) / 2, \quad \mu_{c}\left(b_{k}\right)=0.5$, parameter $c_{k}$ is taken as the value of the OTSU threshold segmentation of intensity maps of various scales, and $c_{k}=T_{k}$, parameter $a_{k}=0.8 T_{k}$. This ensures the membership value is one when the information of the original four-scale changes passed through the fuzzy transformation. The change intensity observation $Y_{k}(i, j)$ with respect to the non-change category $w_{u} \quad$ 's membership $\mu_{u}\left(\mathrm{Y}_{k}(i, j)\right)$ is : $\mu_{u}\left(\mathrm{Y}_{k}(i, j)\right)=1-\mu_{c}\left(\mathrm{Y}_{k}(i, j)\right)$. Apparently, the change intensity observation $Y_{k}(i, j)$ with respect to the change category $w_{c}$ and the non-change category $w_{u}$ 's membership satisfaction is: $0 \leq \mu_{c}\left(\mathrm{Y}_{k}(i, j)\right) \leq 1,0 \leq \mu_{u}\left(\mathrm{Y}_{k}(i, j)\right) \leq 1$. If $\mu_{c}\left(\mathrm{Y}_{k}(i, j)\right)$ 's value is closer to one, the possibility of the intensity of the observed values $Y_{k}(i, j)$ belonging to the change category $w_{c}$ is greater, otherwise, the possibility belongs to nonchange category where $w_{u}$ is greater (Wang et al., 2010). The fuzzy relation matrix of the fourth scales is:

$$
R_{i j}=\left[\begin{array}{llll}
\mu_{c}\left(Y_{1}(i, j)\right) & \mu_{c}\left(Y_{2}(i, j)\right) & \mu_{c}\left(Y_{3}(i, j)\right) & \mu_{c}\left(Y_{4}(i, j)\right) \\
\mu_{u}\left(Y_{1}(i, j)\right) & \mu_{u}\left(Y_{2}(i, j)\right) & \mu_{u}\left(Y_{3}(i, j)\right) & \mu_{u}\left(Y_{4}(i, j)\right)
\end{array}\right]^{T}
$$

In this paper, the adaptive method is used to weigh the results of the individual scales (Wang et al., 2009), and the formula is as follows:

$$
W_{p}=\frac{1}{\sigma_{p}^{2} \sum_{p=1}^{4} \frac{1}{\sigma_{p}^{2}}} \quad \mathrm{p}=1,2,3,4
$$

Where $\sigma_{p}$ is the standard deviation of the change detection results under $\mathrm{p}$ scales, and $W_{p}$ is the weight of $\mathrm{p}$ 's scale change detection results. According to the fuzzy weighted linear transformation:

$$
\mu_{i j}=\left(\mu_{c, i j}, \mu_{u, i j}\right)=W R_{i j}
$$

$\mu_{c, i j}$ and $\mu_{u, i j}$ are four-scale change information after a fuzzy fusion cell $(i, j)$ belonging to the comprehensive possibility of changes category and the non-change category. Based on the maximum membership criterion, when $\mu_{c, i j}<\mu_{u, i j}$, the target object does not change, when $\mu_{c, i j} \geq \mu_{u, i j}$, the target object changes.

\subsection{Decision-level fusion analysis}

Solution 1: The OTSU threshold segmentation is performed on the intensity diagram of the variations on four scales, the changing pixel values and the non-changing pixel values from the test results are respectively represented by 1 and 0 . Through the establishment of a decision-making binary tree shown in Figure 2, fusing the changing detection results of the variations on four scales, and the final result of all the pixels is divided into five categories, as shown in Table 1.

Table.1 Rule of decision-level fusion

\begin{tabular}{cc}
\hline Category & Change Detection Results \\
\hline Class0 & No pixel change occurs on four scales \\
Class1 & Pixel change occurs on one scale \\
Class2 & Pixel change occurs on two scales \\
Class3 & Pixel change occurs on three scales \\
Class4 & Pixel change occurs on four scales \\
\hline
\end{tabular}

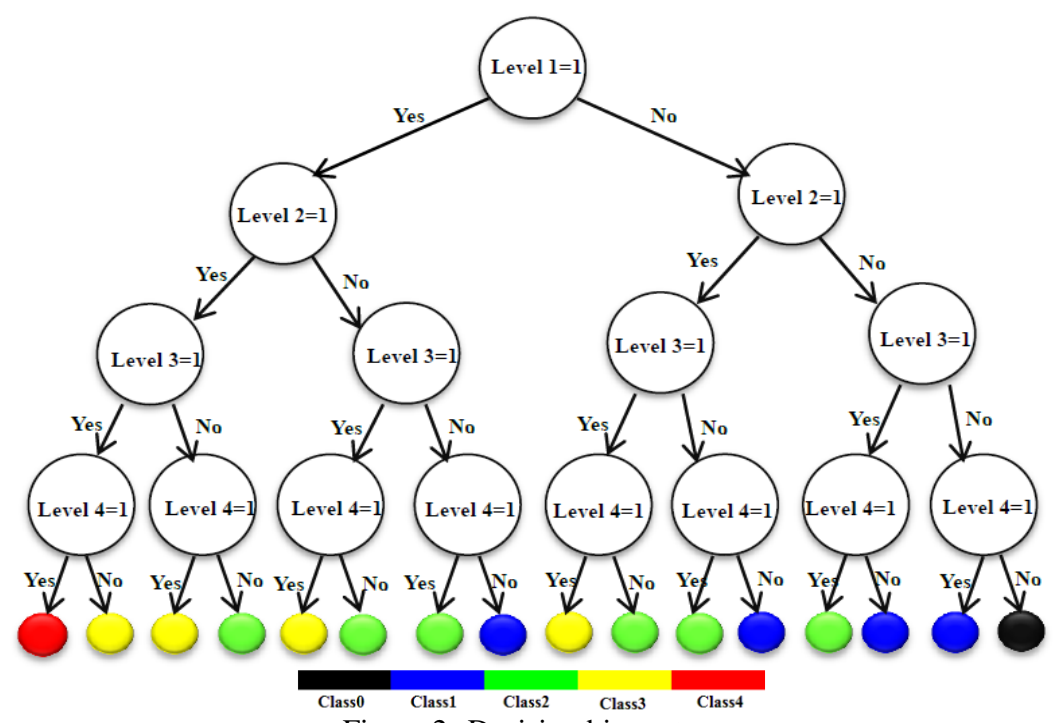

Figure.2 Decision binary tree 
Solution 2: First, we put the change vector modulus of four scale objects in an ascending order. Then use the following exponential function to make a curve fitting of the change vector modulus values of the four scales:

$$
\mathrm{f}(x)=a_{1} e^{b_{1} x}+a_{2} e^{b_{2} x}
$$

in the formula above $a_{1} 、 b_{1} 、 a_{2} 、 b_{2}$ are fit factors.

In this paper, according to the four-scale fitting function, which defines the following changes in intensity in order to quantify the change in the difference between $-2 \sim 2$, using the following piecewise function, assuming the average step size is $\Delta=\frac{\max -\min }{5}$, in which $\max =\max (\mathrm{f}(\mathrm{x}))$, $\min =\min (\mathrm{f}(\mathrm{x})), \quad x \in\left[1\right.$, num $\left._{c}\right]$ and $\mathrm{x}$ is an integer, num $_{c}$ means the total number of spots on the 4 scales respectively. Then quantized value $\mathrm{W}$ is:

$$
W=\left\{\begin{array}{cc}
-2 & Q_{c} \in[\min , \min +\Delta] \\
-1 & Q_{c} \in[\min +\Delta, \min +2 \Delta] \\
0 & Q_{c} \in[[\min +2 \Delta, \min +3 \Delta]] \\
1 & Q_{c} \in[\min +3 \Delta, \min +4 \Delta] \\
2 & Q_{c} \in[\min +4, \max ]
\end{array}\right.
$$

In which, the unchanged(Unchanged, UC) regional value is -2 , the faint change (Faint Change, FC) regional value is -1 , the medium change (Medium Change, MC) regional value is 0 , the obvious change (Obvious Change, OC) regional value is 1 , the strong change (Strong Change, SC) regional value is 2 (Wu et al., 2013), in order to integrate the changes in the results of the various scales. In this paper, the multi-valued logic is drawn to establish decision fusion rules (Chen et al., 2002). First, integrate the scale of both Level 1 and Level 3 test results into a change in the scale of intensity map, then integrate the scale of Level 2 and Level 4 test results into another change in the scale of intensity map. Finally, the intensity of these two changes are integrated in order to facilitate the integration of the location and the intensity of the change.

The decision level fusion rule corresponds to a five valued logic function $F(x, y)$, Figure 3 is $F(x, y)$ and $x$, $y$ 's relational graph. The decision level fusion rule designed in this paper is shown in Figure 4, below. When the intensity of the change of the two scales is large, then the intensity of the fusion should be greater; when the intensity of the change of the two scales is small, then the intensity of the fusion should be smaller. In the figure above, $\mathrm{F}(\mathrm{x}, \mathrm{y})$ 's the overall trend is increasing, with the increase of $x+y$ value, for example: when $\mathrm{F}(\mathrm{x}, \mathrm{y})=2$, then $\mathrm{x}+\mathrm{y}=3$ or $\mathrm{x}+\mathrm{y}=4$, it means among $\mathrm{x}$, there is at least one area that is strongly changing, another one is an obvious change region, where the fusion rules defining synthetic strength should be as intensely changing, so as to fully reflect change information in the strength of the two scales (Wu et al., 2013).

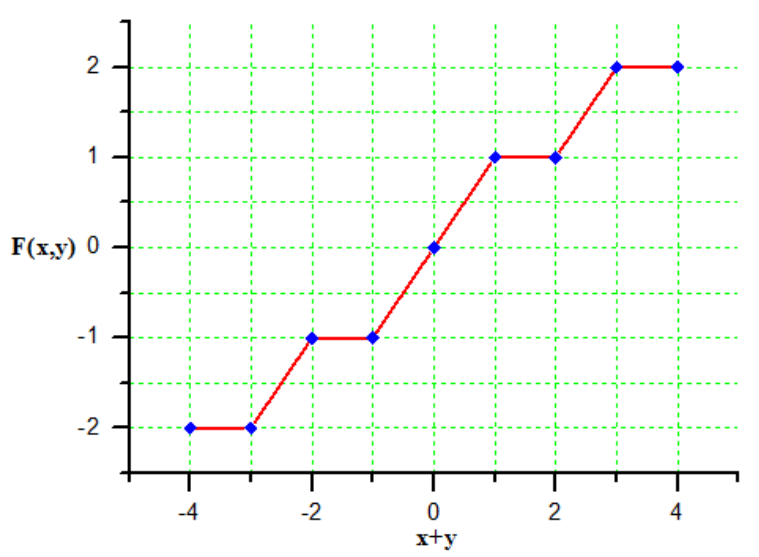

Figure.3 Logical function F(x, y)

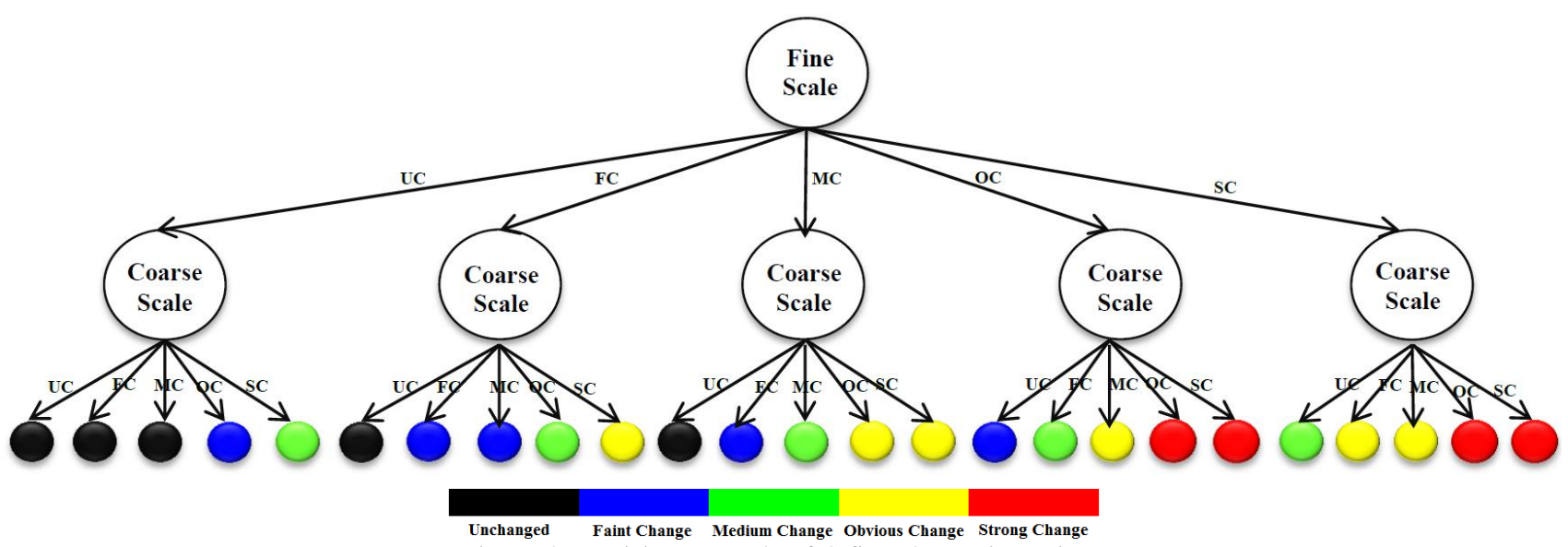

Figure.4 Decision tree rule of define change intensity

\section{EXPERIMENTAL RESULTS AND ANALYSIS}

\subsection{Test Data}

The remote sensing image data of the change detection test used in this paper is the multi-spectral image of SPOT5 in a district of Guangzhou city in 2006 and 2007. It contains three bands of red, green and blue, with the resolution of $2.5 \mathrm{~m}$, an image size of 916 pixels $\times 1096$ pixels, as shown in Figure 5. The features of the two phase images are rich, and the test area mainly consists of 6 kinds of features, including paddy fields, bare land, buildings, roads, settlements and greenery. The resolution of the image is relatively high. If the multi-scale segmentation scale is appropriate in the process, then the segmented polygons are able to draw a clear outline of objects, and segmentation of objects are not too broken (Wu et al., 2013). In this paper, eCognition software is used to obtain corresponding object, and the two temporal 
images are unitedly segmented. This step is called united segmentation. Then, focusing on the different size of objects, such as roads, paddy fields, building roofs, settlements, green field, etc., we choose multiple segmentation scale. The best classification principles "the homogeneity of the same class is keep largest, while the heterogeneous of different class is keep largest" is used to explore these specific categories of best feature segmentation scale.

The improvement is made on the basis of the method proposed by Zhai and Zhang et al. (Zhai et al., 2011; Zhang et al., 2009). The weights of the multi-band image segmentation are introduced into the calculation of the ratio of mean difference to neighbors(ABS) to standard deviation (ratio of mean difference to neighbors(ABS) to standard deviation, RMAS) (Zhang et al., 2009), RMAS is the ratio of the object and the neighborhood average absolute difference value between the target standard deviation, the improved formula is:

$$
\left.\begin{array}{c}
R M A S=t_{L} \bullet \frac{\Delta C_{L}}{S_{L}} \\
S_{L}=\sqrt{\frac{1}{n-1} \sum_{i=1}^{n}\left(C_{L i}-\overline{C_{L}}\right)^{2}} \\
\Delta C_{L}=\frac{1}{q} \sum_{i=1}^{u} q_{s i}\left|\overline{C_{L}}-\overline{C_{L i}}\right|
\end{array}\right\}
$$

in the formula above, $\mathrm{L}$ is the number of bands in the image, $t_{L}$ is the weight of the band during segmentation, $\Delta C_{L}$ is the absolute value of single-band objects and the ratio of mean difference to neighbors, $S_{L}$ is the standard deviation of the image in a single wave segment, $C_{L i}$ is the i pixel gray value on $\mathrm{L}$-band, $\overline{C_{L}}$ is the average value of the concerned image objects, $\mathrm{n}$ is the number of pixels inside of image objects, $u$ is the number of objects directly adjacent to the target object, $\mathrm{q}$ is the target object boundary length, $q_{s i}$ is the common boundary length of target object with the i-th directly adjacent objects, $\overline{C_{L i}}$ is the layer mean value of the i-th directly adjacent objects (Zhang et al., 2009).

When the segmentation scale is small, the object has the same object, the $S_{L}$ value of the object is small, adjacent objects of $\mathrm{L}$ belong to the same category and have a strong spatial dependence. At this time, the value of $\Delta C_{L}$ and the value of RMAS objects are small; When the segmentation scale is close to the class object, the object contains the same surface features, and the $S_{L}$ value of the object is small. Adjacent objects belong to different classes leaving the spatial dependence weakened, at this time, the $\Delta C_{L}$ of the object and RMAS values are larger; When segmentation scale is further increased, an object will contain other types of surface features, the $S_{L}$ value of the object becomes larger, the spatial dependence with neighborhood objects will increase, $\Delta C_{L}$ of the object and RMAS values begins to decrease at this time (Zhang et al., 2009). During the experiment, on each scale for each category of feature selected a certain number of samples to calculate the RMAS value of the specific surface features on different scales, and take the RMAS value of the object on the Y-axis, the image segmentation scale value on the $\mathrm{X}$-axis, drawing the line chart of segmentation scale change with different objects of the RMAS value, as shown in Figure 6. At last, the optimal scale of information extraction of different objects can be selected by the RMAS line chart of the image object.

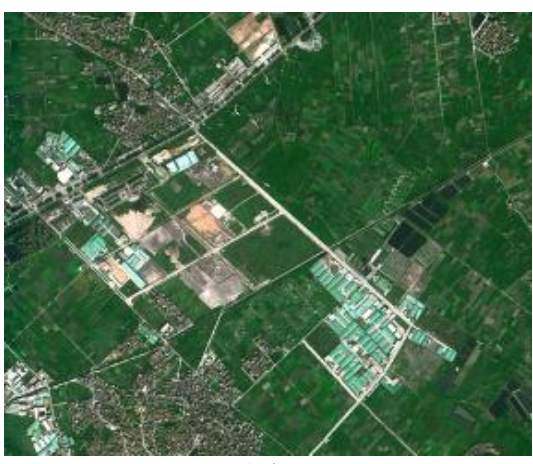

(a)

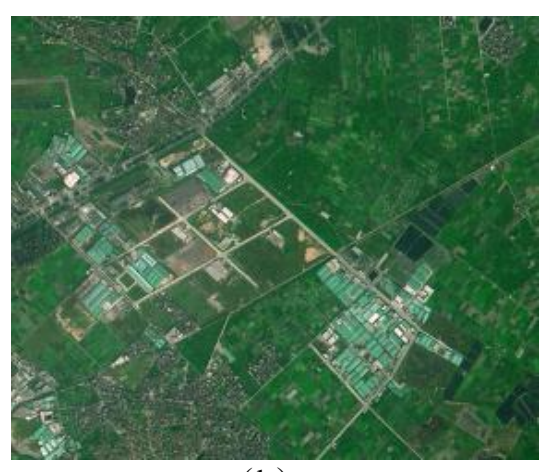

(b)

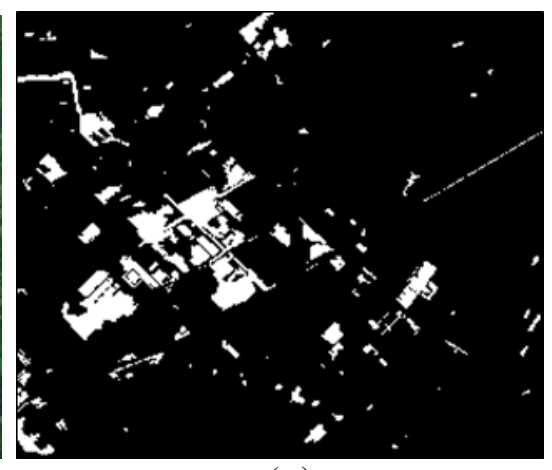

(c)

Figure.5 Dataset used in the experiment. (a) Remote sensing image of year 2006 (b) Remote sensing image of year 2007 (c) Reference change detection image

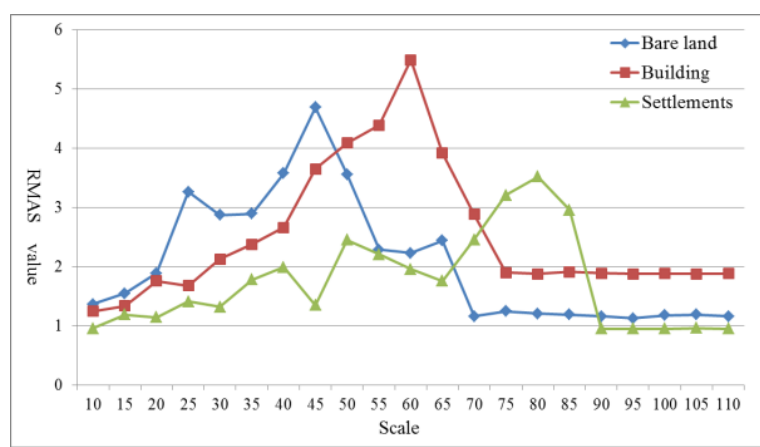

(a)

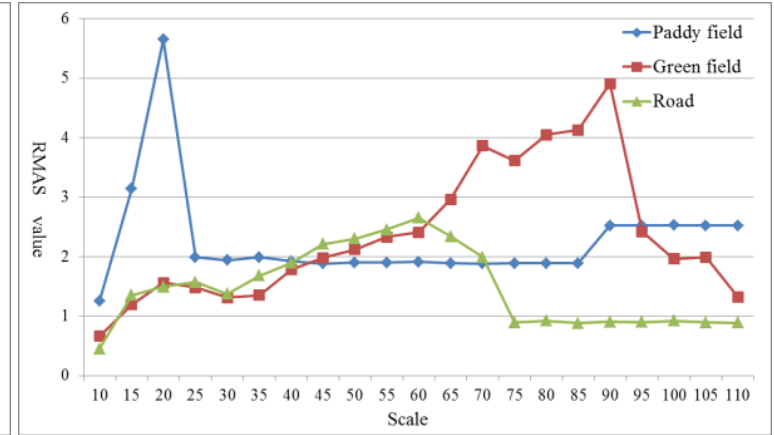

(b)

Figure.6 The line chart of RMAS 


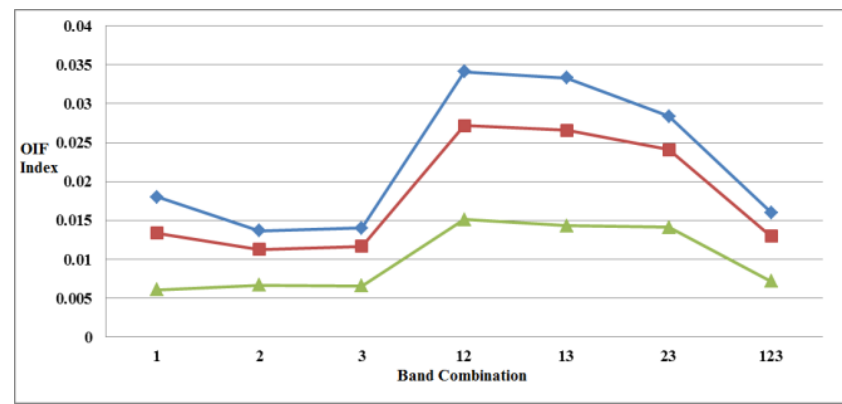

(a)

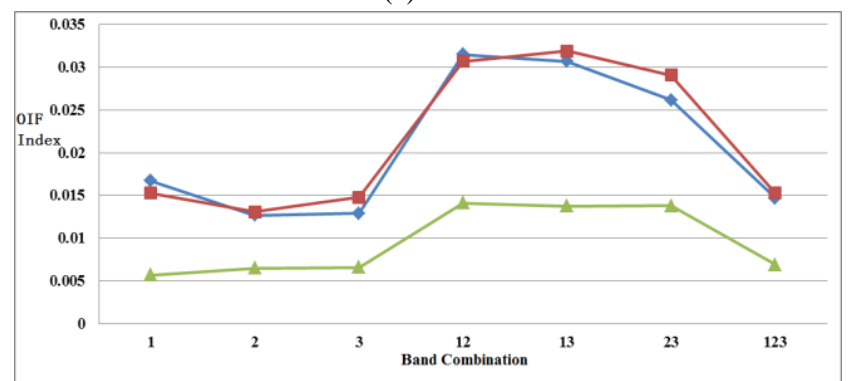

(c)

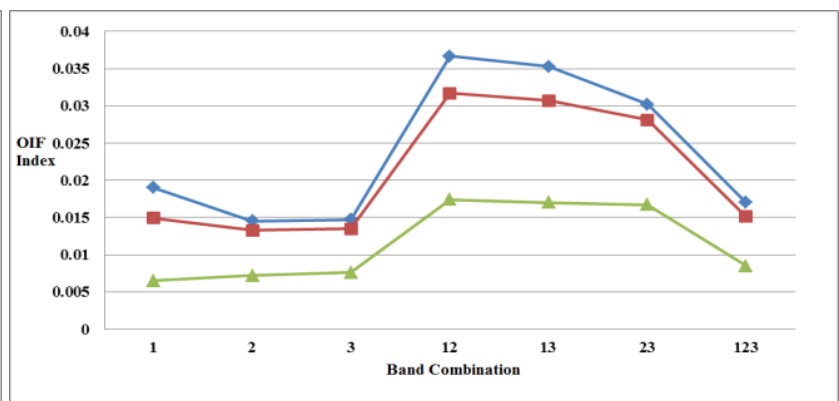

(b)

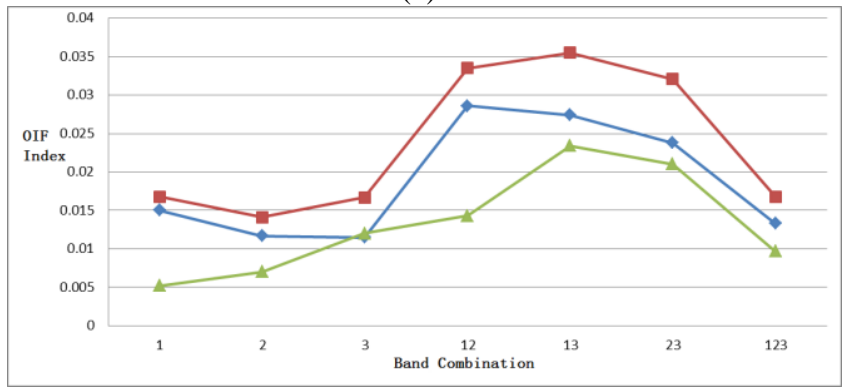

(d)

$\rightarrow$ Mean $\rightarrow$-Standard deviation $\rightarrow$-GLCM Entropy

Figure.7 The sketch map of OIF index

It can be seen from Figure 6 that: the optimal segmentation scale of paddy fields, bare land, buildings, roads, settlements, and greenery are $20,45,60,60,80$ and 90 respectively. Besides, as can be seen from the line graph of RMAS, some of the features of the surface will appear a plurality of local peaks, which is further explains that the optimal size for each object is relative, usually within a value range. This paper selects the segmentation results of four scales, Level1, Level2, Level3, Level4 (scale20, 45, 60, 80 ), to conduct the best band calculation of spectrum and texture feature differences, the results are shown in Figure 7.

According to Figure 7(a), (b), the spectral mean value and the texture entropy difference, the OIF index of single band is lower, and the overall of OIF index is higher when there are two bands, when band 1, 2 combine, the OIF index is at its peak. With the increasing number of bands, the OIF index shows a downward trend, when the band 3.2 Change detection results and accuracy evaluation

In order to verify the feasibility and effectiveness of the three fusion strategies proposed in this paper, by using SPOT5 multi-spectral image data sets, with rich variation of image features of both types, the changes of the two images features are rich. Moreover, within the coverage area of the image, a typical feature is mainly composed of agricultural land, settlements, bare land and buildings, through different spectral changes feature in two images, the main changes can be observed as the conversion of agricultural land and bare land, between buildings and between different bare land.

The results of the three fusion strategies are shown in Figure 8. In which, Figure 8(a) is fuzzy fusion test results for four scale change information; The results of the change detection are shown in Figure 8 (b) by using decision level fusion program, the final result figure includes five categories

Finally, the method of multi-scale integration and the conventional pixel-based change detection method such as Change Vector Analysis (CVA), principal component analysis (PCA), independent principal component analysis (ICA), the results of the correlation coefficient method which have been adopted in this paper are compared. Precision index test combination is 123 , the OIF index is significantly reduced. This shows it is not the greater the number of bands, the greater the information. Similarly, in Figure 7 (c), when the spectral mean value and the texture entropy difference combine at band 1,2, the OIF index is at its peak, but when the spectral standard deviation difference combine at band 1,3, the OIF index is at its peak; In Figure 7(d), when the spectral mean value combines at band 1,2 , the OIF index is at its peaks, however, when the spectral mean value and the texture entropy difference combine at band 1,3. OIF indexes were applied in four scales respectively, to look for the best band combination between spectrum and texture difference, then by constructing the difference vector of the object, using the method of vector analysis based on objects to construct the difference image of four dimensions.

of pixels; Decision level fusion proposal 2 is used to detect the changes of the results as shown in Figure 8 (c), the final result maps not only reflect the change of the position information of the pixels, but also reflect the information of the intensity of its change. In order to compare the results of two kinds of decision-level fusion methods and the results of fuzzy fusion, the unchanged pixel (Class 0) in Figure 8 (b) and the change in pixel (Class 1) only occurs on one scale are considered not to be changed, the remaining pixels are considered to be changed, then the decision level fusion method of binarization results are shown in Figure 9 (a). Likewise, if one considers the unchanged area and slight changed area in Figure 8 (c) as no change, the rest is seen as the area of change, then the decision level fusion method of binarization result is shown in Figure 9 (b). results are shown in Table 2 below. Since the pixel-based the correlation coefficient change detection result is superior, this paper only gives the results of correlation coefficient. As shown in Figure 9(c), it can be seen that the correlation coefficient appeared very susceptible to the phenomenon of "salt and pepper", the main reasons for analyzing are the 
method of image registration accuracy, the radiation correction with higher requirement depending on the selected threshold at the same time; Moreover, the noise of the image has a greater impact on the change detection results. Table 2 shows the change in the detection accuracy of the table, which can be seen in the proposed three multi-scale fusion policies of this paper, among them, the overall accuracy, false detection rate, the missing rate, and the Kappa coefficient are better than those of conventional detection methods based on the change of pixels.

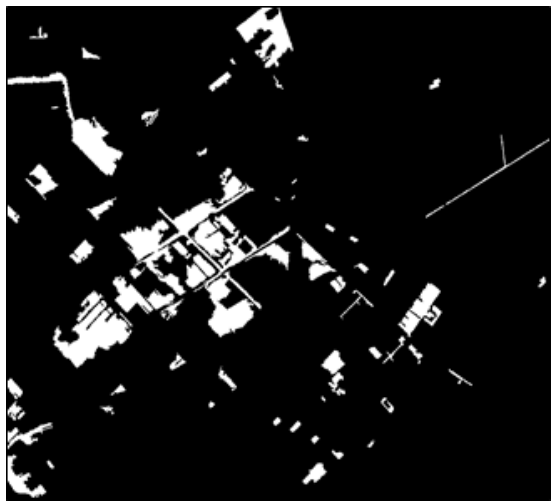

(a)

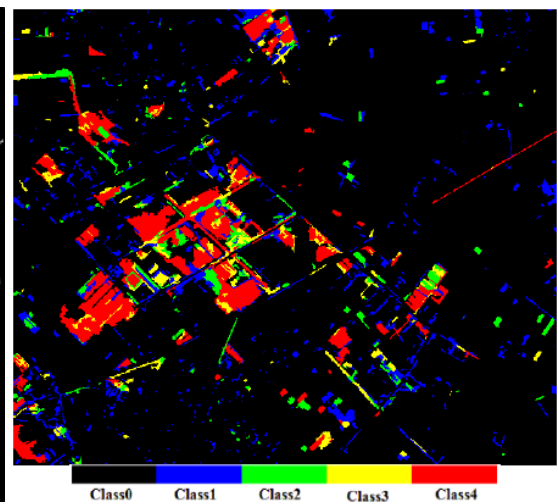

(b)

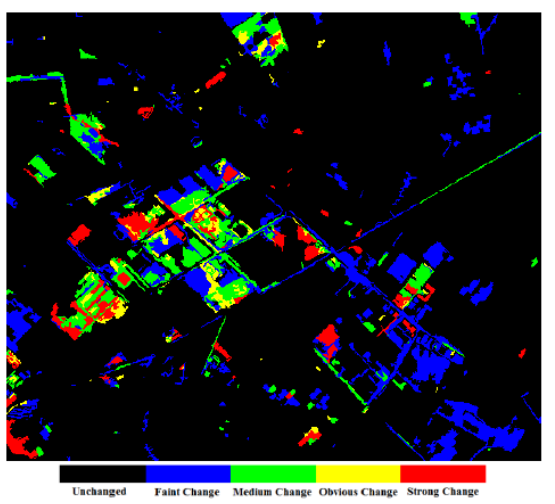

(c)

Figure.8 The results of Multi-scale fusion (a) Fuzzy fusion (b) Decision level fusion 1 (c) Decision level fusion 2

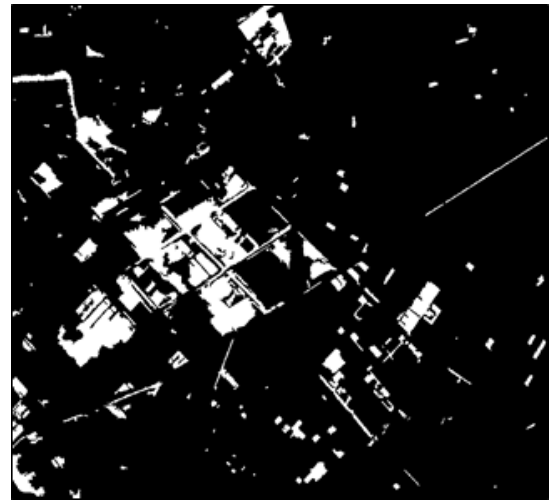

(a)

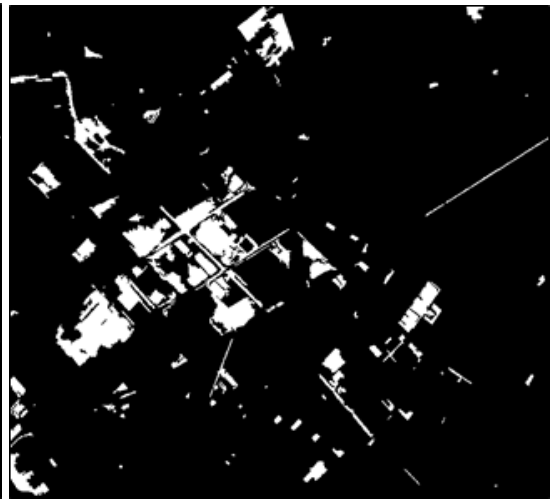

(b)

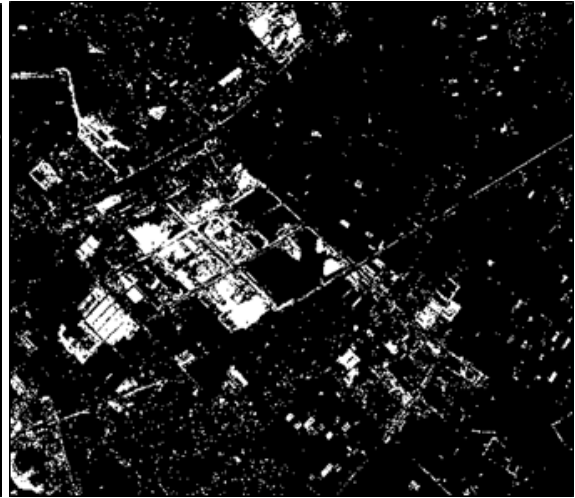

(c)

Figure.9 The results of change detection (a) Decision level fusion 1 (b) Decision level fusion 2 (c) Correlation coefficient method based on the pixels

Table.2 Precision of change detection

\begin{tabular}{|c|c|c|c|c|c|}
\hline & Methods & $\begin{array}{c}\text { Overall accuracy } \\
(\%)\end{array}$ & $\begin{array}{l}\text { False alarm rate } \\
\qquad(\%)\end{array}$ & $\begin{array}{l}\text { Miss detection rate } \\
(\%)\end{array}$ & Kappa index \\
\hline \multirow{4}{*}{ Pixel-based } & CVA & 75.45 & 48.50 & 32.61 & 0.4992 \\
\hline & PCA & 78.23 & 45.40 & 29.10 & 0.5493 \\
\hline & ICA & 78.76 & 44.72 & 29.74 & 0.5518 \\
\hline & Correlation Coefficient & 82.34 & 43.34 & 28.61 & 0.5602 \\
\hline \multirow{3}{*}{ Object-based } & Fuzzy fusion & 90.81 & 13.87 & 12.50 & 0.8202 \\
\hline & Decision level fusion 1 & 90.75 & 13.31 & 16.73 & 0.8120 \\
\hline & Decision level fusion 2 & 91.15 & 13.15 & 13.53 & 0.8211 \\
\hline
\end{tabular}

From Table2 we can see that:

(1)The variation test accuracy of the 3 kinds of multi scale fusion methods is better than that of pixel-based methods. The overall accuracy of fuzzy fusion and two decision level integrated method with the Kappa coefficient equal to $90.81 \%, 0.8202,90.75 \%$, and $0.8120,91.15 \%$ and 0.8211 . The overall accuracy of three kinds of methods compared with the pixel-based methods has improved by $10 \%$ or so. The Kappa coefficient based on the picture element method belongs to 0.4 06, and the Kappa coefficient of the three kinds of methods kept between 0.8 and 1 , and the effect is improved greatly.

(2)The results of the 3 kinds of multi - scale fusion methods have different advantages. Because of the relatively small size of the segmentation results, the smaller area of ground objects is better, and the settlement group, agricultural land and other large objects can be used in the coarse scale, the fuzzy fusion method is applied to the calculation of the variance of the 4 scales. The arrangement order of the weight is $0.165,0.206,0.261,0.368$, and the results of the final results show that the information about the variation of the information on the 4 scales is effective and accurate. The data of the detection accuracy of Table 2 is confirmed. Decision level fusion scheme 1 is dependent on the variation of the intensity of each scale. The change of the 4 scales is simple, and the change of the pixels in the two scales and two scales above is considered the final change. The rest are not changed. Compared with the fuzzy fusion 
method, the false detection rate is reduced, but the false detection rate is large, and the overall accuracy is slightly lower than that of the fuzzy fusion method. Therefore, in practice, if the fusion scale selection is not appropriate or the results of the threshold segmentation are not good, they will the greater impact on the final fusion effect.

(3) Decision level fusion scheme 2 is used to quantify the difference between 5 kinds of changes in the process of threshold segmentation. The decision level fusion rule is built by using multi-valued logic functions. The final result not only reflects the position information of the pixels, but also

\section{CONCLUSIONS}

In this paper, a novel change detection method based on multi-scale segmentation and fusion is proposed. The method is effective due to the integration of information on multiple scales. Compared with the traditional pixel level change detection method, the three types of fusion strategies are adopted to achieve better results which also prove the feasibility and effectiveness of the fusion strategies. After extracting the change area, this paper does not identify the reflects the information of its intensity. Moreover, the overall accuracy of the scheme 2 is the highest, the false positive rate and the missed detection rate are better than scheme1.

(4) In addition, based on the surface measurement, the image change detection node fruit area is relatively regular, and most of the objects correspond with actual physical meaning in the target region, and in pixel level change detection results there exists a large amount of salt and pepper noise, and the region is broken, which result in the whole medical test results being poor.

category of it. These works will continue to be improved in future research.

\section{ACKNOWLEDGEMENT}

This work was supported by National Key Fundamental Research Plan of China (973) [Project NO. 2012CB719906], National High Technology Research and Development Programme of China (863 Programme) [Project NO. 2013AA122301]

\section{REFERENCES}

Su X., Wu W., Li H., et al. 2011. Land-Use and Land-Cover Change Detection Based on Object-Oriented Theory. International Symposium on Image and Data Fusion (ISIDF).Tengchong:IEEE,1-4.

Hazel G. 2001.Object-level change detection in spectral imagery. IEEE Transactions on Geo-science and Remote Sensing, 39(3):553-561.

Dai Q., Liu J., Liu S. 2012. Remote Sensing Image Change Detection Using Particle Swarm Optimization Algorithm. Acta Geodaetica et Cartographica Sinica,41(6):857-860.

Liang H. 2013. Classification of high resolution remote sensing image based on Geo-ontology and Conditional Random Fields. Proceedings of SPIE 8921, MIPPR 2013:Remote Sensing Image Processing, Geographic Information Systems, and Other Applications. Wuhan: SPIE.

Zhong J., Wang R. 2005. Multi-temporal Remote Sensing Image Change Detection Based on Adaptive Parameter Estimation. Acta Geodaetica et Cartographica Sinica, 34(4):331-336.

Wang C., Xu M., Wang X., et al.2013. Object-oriented change detection approach for high-resolution remote sensing images based on multi-scale fusion. Journal of Applied Remote Sensing, 7(1):073696.

Sun K., Chen Y. 2010. The Application Of Objects Change Vector Analysis In Object-level Change Detection. International Conference on Computational Intelligence and Industrial Application (PACIIA) ,15(4): 383-389.

Bovolo F., Bruzzone L. 2007. A theoretical framework for unsupervised change detection based on change vector analysis in the polar domain. IEEE Transactions on Geo-science and Remote Sensing , 45(1):218-236.

Bruzzone L., Prieto D. 2000. Automatic analysis of the difference image for unsupervised change detection. IEEE Transactions on Geoscience and Remote Sensing, 38(3):1171-1182.

Nemmour H., Chibani Y.2006. Multiple support vector machines for land cover change detection: an application for mapping urban extensions. ISPRS Journal of Photogrammetry and Remote Sensing, 61(2):125-133.

Wang W., Zhao Z., Zhu H.2009. Object-oriented Multi-feature Fusion Change Detection Method for High Resolution Remote Sensing Image. Proceedings of the $17^{\text {th }}$ International Conference on Geo-informatics. Fairfax, VA:IEEE,1-6.

Wu J., Yan W., Ni W., et al.2013. Object-Level Change Detection Based on Image Fusion and Multi-Scale Segmentation. Electronics Optics \& Control, 20(12): 51-55.

Su J., Liu D.2007. An Object level Change Detection Algorithm for Remote Sensing Images. ACTA PHOTONICA SINICA, 36(9):1764-1767.

Chen C., Wu G.2011. Evaluation of Optimal Segmentation Scale with Object-oriented Method in Remote Sensing. Remote Sensing Technology and Application, 26(1):96-101.

Li L., Shu N., Wang Y. 2011. Segment-based Remote Sensing Image Change Detection Using Normalized Mutual Information. Remote Sensing Information(Theoretical research), (6): 18-22.

Liang J., Yang J., Zhang C.,et al. 2012. A Comparison of Two Object-Oriented Methods for Land-Use/Cover Change Detection with SPOT5 Imagery. SENSOR LETTERS, 10(1-2):415- 424.

Wu W., Liu Z. 2007. A method of the best band combination selection based on spectral characteristics of surface features. Engineering of Surveying and Mapping ,16(6):22-24.

Zhai Y., Wang Y., Zhang S. 2011. An optional scale segmentation method based on scale comparison in object-oriented remote sensing classification. 2011 International Remote Sensing, Environment and Transportation Engineering (RSETE), Nanjing:IEEE:4752-4755.

Zhang J., Wang Y., Li Y., et al. 2009. An Object-Oriented Optimal Scale Choice Method for High Spatial Resolution RemoteSensing Image. Science and Technology Review,27(21):91-94.

Tobias O J., Seara R. 2011. Image Segmentation by histogram thresholding using fuzzy sets. IEEE Transaction on Image Processing, 11(12):1457-1465.

Wang G., Wang Y., Jiao L. 2010. Change Detection Method of Multi-band Remote Sensing Images Based On Fast Expectation- 
Maximization Algorithm and Fuzzy Fusion. Journal of Infrared Millimeter Waves, 29(5): 384-388.

Wang W., Zhao Z., Zhu H. 2009. Object-oriented Change Detection Method Based on Multi-scale and Multi-Feature Fusion. Joint Urban Remote Sensing Joint Event. IEEE:1-5.

Chen H., Shen J. 2002. Canonical form of fuzzy logic function based on multi-valued logic method. Journal of Zhejiang University:Science Edition, 29(3):292-296. 